most often adopted. Some temporary amelioration of the symptoms and control of spasm was mentioned in 2 cases, but paralysis of the legs, lasting 24 hours, was noted in another.

Sedative drugs have been used in the great majority of cases. In order of frequency these were as follows: Chloral, bromide, morphia, chloretone, atropine, omnopon, scopolamine, alcohol, chloroform, and paraldehyde. In some cases, though but few, it was noted that one or other of these drugs had been useful in controlling spasm.

Although fully sympathising with the natural desire of the busy reader to find at the end of a paper such as this a series of "conclusions," we have abstained from them, being very conscious that we have much more to learn on every point under discussion. However, we have not hesitated to express our own views on a number of these points as we have dealt with them, and we trust that they may at least serve to rouse further interest in the specific treatment of tetanus, which we believe to be of greatest value, did we but know how to apply it aright.

\section{MODIFIED TETANUS.}

BY H. BURROWS, M.B., B.S. LoND., F.R.C.S. ENG., captaIN, R.A.M.C. (T.F.).

THERE are two reasons why the subject of tetanus should be of interest at the present moment. In the first place, the disease still occurs amongst the wounded. During the months of July, August, and September, 1916, at the

General Hospital we had one case of tetanus in every 600 cases of gunshot wound. And this, of course, does not represent the full liability, for cases have occurred in patients who have been evacuated to England, and possibly at casualty clearing stations also. In the second place, a large proportion of the cases which have been seen recently have been abnormal in character, inasmuch as the muscular spasms have not become general. They have remained localised to the muscles in the neighbourhood of the original wound. Moreover, apart from the restriction of symptoms to the muscles in the vicinity of the wound, there are certain other characters which differentiate local or modified tetanus from the natural variety. These will receive attention later. In the meantime it may be remarked that in local or modified tetanus we have a new form of disease. The earliest record of a case ${ }^{1}$ is now little more than three years old. The disease is new because its cause is new, for local tetanus is tetanus modified by the prophylactic use of antitetanus serum,

Forms of modified tetanus.-Cases of modified tetanus may be considered in three groups ${ }^{2}$-namely: (1) splanchnic tetanus ; (2) cephalic tetanus ; (3) local tetanus of the limbs. In all of these forms general spasms with opisthotonos are absent. Cases have been recorded which do not fall into any of these groups-for example, tetanus localised to the abdominal muscles. Nevertheless, these three groups will cover most of the ground and will provide sufficient material for discussion.

\section{Splanchnio Tetanus.}

Splanchnic tetanus ${ }^{3}$ follows upon lesions of the viscerathat is to say, penetrating wounds of the abdomen and thorax. It is nearly always fatal and the course is exceedingly rapid, so that death ensues within 24 or 48 hours from the onset of symptoms. Opisthotonos and general convulsions do not occur, the spasms being limited to the muscles of deglutition and respiration. The difficulty in swallowing is a pronounced feature, and may be so severe that the case resembles one of hydrophobia, in which case the sight, or even the mere mention, of a glass of water causes the severest pharyngeal spasm, accompanied usually by acute dyspnœa.

\section{Cephalic Tetanus.}

Of head tetanus there are four varieties : (1) Non-paralytic ; (2) the form accompanied by facial paralysis; (3) that accompanied by oculomotor paralysis; and (4) a form in which paralysis of the hypoglossal nerves is a special feature. Of these, the non-paralytic form is said to be the least common, ${ }^{4}$ some motor paralysis being the rule in cases of head tetanus. And, as may be expected from its rela. tively wide distribution, the facial nerve is the nerve that is most commonly affected.
Head tetanus with facial paralysis. - This variety follows a wound of the face or its close neighbourhood, and is characterised by: (1) Unilateral trismus, which may become bilateral, and later on (2) difficulty in swallowing; (3) facial paralysis on the same side as the wound. The most remarkable feature is the fact that spasms occur in the paralysed muscles of the face. The explanation of this phenomenon is that there is an alteration in the conductivity of the peripheral branches of the facial nerve, which results in a partial nerve-block, sufficient to prevent the passage of voluntary impulsts but insufficient to block the more powerful and pathological impulses of tetanus. The facial paralysis may persist after the other symptoms have disappeared, but ultimately, as a rule, the face recovers.

Hypoglossal form.-This form appears to be rare. As a matter of fact, in Major Kazanjian's Jaw Clinic there has never been a case, although the wounds treated there are of the kind in which hypoglossal tetanus might be anticipated.

Ophthalmoplegio form.-Ophthalmoplegic tetanus follows wounds of the orbit or its immediate neighbourhood. Ptosis is said to be the commonest symptom, but any or all of the ocular muscles, extrinsic or intrinsic, may be paralysed.

\section{Local Tetanus of the Limbs.}

The last abnormal form of tetanus is that which is limited to one or more of the limbs-that is to say, to one arm, or to one leg, or to both legs. These have been described as the monoplegic and paraplegic types. I do not know whether tetanus limited to the two upper extremities ever occurs. And as regards the simultaneous affection of one arm and one leg-a hemiplegic form-it is difficult to see how this could come about except as the result of simultaneous lesions of these two members.

The chief symptoms of local tetanus are clonic and tonic spasms of the wounded limks. The clonic spasms have been described in some of the recorded cases as being accompanied by considerable pain, and as lasting for four stconds. As a matter of fact, in the four cases we have had at the

General Hospital the clonic movements have been painless and have been mere twitchings, lasting, perhaps, for balf a second each. In three out of the four cases these twitchings were the first symptoms to be noticed, while in the fourth case the patient complained that his arm was stiff, and it was found that spasticity and twitchings were both present. In the two cases which survived the twitchings gradually became less frequent with the passage of time, while the spasticity became more pronounced and showed no sign of passing off so long as the patients remained under our observation.

Tetanic spasticity. - In the two fatal cases no tonic spasm at all was observed. It is quite possible that in some cases twitchings may not occur, or may escape observation, and the spasticity may then be the only material result of the tetanus. And unless attention be directed to this possibility, it is likely that the true cause of the tetanic spasticity may be overlooked, while in some recorded cases the tonic spasm has preceded the twitchings, and from these facts it is recommended that "persistent hardness in a recently wounded limb should suggest tetanus." The most likely error is to mistake tetanic rigidity for hysterical spasm, which it closely resembles. But in this connexion it is to be noted that tetanic spasticity does not pass off during sleep-at least it did not so pass off in our cases.

There is a special reflex which is indicative of tetanus, and which may be of help in diagnosing certain cases. This reflex consists of extension of the foot and leg when the sole is stimulated, and is in contrast with the normal reflex, which is a withdrawal of the foot from the stimulus. The knee is placed is a position of slight flexion while the stimulus is applied to the sole. There is another sign which Lieutenant B. S. Simmonds has pointed out, and which is elicited as follows: Suppose the leg to be affected; light touches, even the lightest with a wisp of cotton-wool, will produce reflex spasms in that leg. To bring about the spasm by stimulating the thigh, a more vigorous application is necessary; for example, tapping the skin with the fingers. And the further away from the affected part that the stimulus is applied, the more vigorous must it be to evoke reflex spasms. I have thought that respiration of automatic type is an early sign, and believe that it may precede even trismus, and be the first positively recognisable evidence of the onset of tetanus. 
Post-tetanio anasthes a. - In the two non-fatal cáses of local tetanus of the limbs which we have had at the - Generdl Hospital areas of cutaneous anæsthesia remained. The question arises as to whether this anæsthesia is a consequence of the disease apart from gross trauma of the peripheral nerves. In our caves, the wounds were deep wounds and the cutaneous anæsthesia may have been due to direct injury of a nerve in the wound area. This important point can be cleared up only by further careful observation of cases of local limb tetanus when direct injury of nerves supplying anæsthetic areas can be excluded.

\section{Notes of Cases.}

The following brief notes on the cases of abnormal tetanus which we have had at the —_General Hospital may be of interest. They are six in number, and include one case of splanchnic tetanus, one of head tetanus, and four of tetanus localised to the limbs.

CASE 1. Splanchnic tetanus.- The patient was an officer who had been wounded on Sept. 15th in the abdomen. Laparotomy had been performed and some perforations of the small intestines had been sutured. 500 units of antitetanus serum had been administered. We admitted him on Sept. 23rd, when the abdominal wound was cleanly healed. Three days later, 11 days after receipt of the wound, at 5 P.M. he complained of having a stiff jaW. Definite trismus was present and pharyngeal spasm was a pronounced feature. He rapidly became worse and died at 1.30 next morning-that is to say, $8 \frac{1}{2}$ hours after the first symptoms had been noted. He

had no opisthotonos or general spasms. Death was preceded by unconsciousness and stertorous breathing.

CASE 2. Cephalic tetanus. - The patiènt was wounded on Sept. 25 th by a fragment of shell, which entered his face just above the left temporo-maxillary joint, penetrated the temporal bone, and passed upward into the temporo-sphenoidal lobe of his brain. On admizsion he had aphasia and weakness of the right upper extremity. On the 30th his skull was opened and the foreign body was extracted. Six days later -that is to say, 11 days after being woundedtetanus developed. 750 Photographs of a Patient Illustrating Tetanic Spasticity.

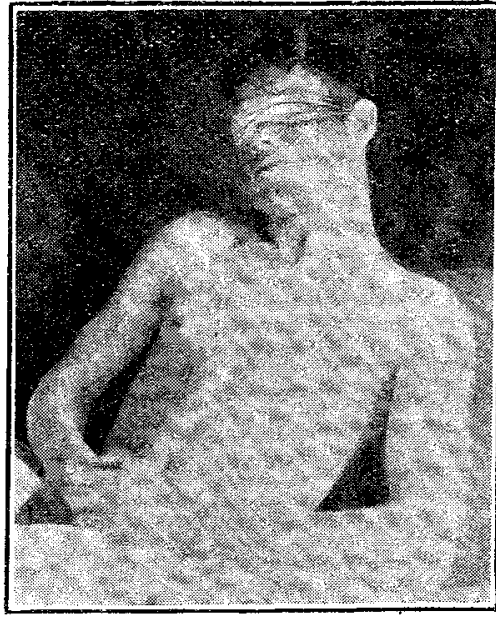

Front view.

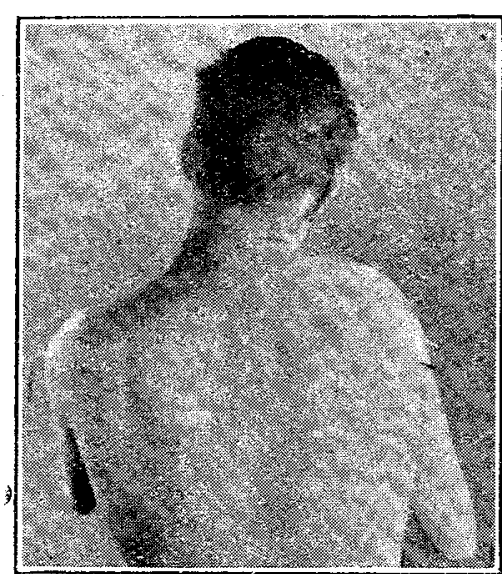

Back view. days later the patient died. Since the amputation the twitchings had continued in the right foot and leg. He never had any trismus or opisthotonos.

CASE 5.-An officer had been wounded slightly in the face on July lst, and had been given antitetanus serum on the following day. He was again wounded by fragments of shell in the calf of the left leg and the left tbigh ten days later. On this occasion no serum was given. On the $25 \mathrm{th}, 14$ days after the second wound, the left foot and leg began to twitch. During the spasm there was plantar flexion of the foot, dorsal flexion of the toes, and flexion of the knee. The twitchings were painless. Gradually the affected muscles became spastic so that a week after the onset they no longer became relaxed between the spasms. The twitchings, as time went by, became less obvious, until they were no longer noticeable, but the tetanic rigidity remained unaltered up to the time of the patient's evacuation to England on Sept. 9th, three months after the receipt of his wound. There was anæsthesia to light touch of the heel and inner side of the foot.

CASE 6.-This patient is the subject of the illustrations. (See figures.) He had been wounded on Jily $23 \mathrm{rd}$ in the face and back, where there was an entry wound at the vertebral end of the spine of the right scapula. There was no exit wound. He had been given antitetanus serum on the day following the receipt of his wound. On August 14th, 22 days after being wounded, he complained of stiffness of the right arm, which was then observed to be twitching and to be spastic, with the arm slightly abducted from the trunk and the elbow flexed. The spasm could be overcome by steady traction until the arm was fully extended without causing the patient any pain, but as soon as the traction ceased the arm resumed its spasmolic position. In the course of the next few days the right trapezius and sterno-mastoid were observed to be twitehing and to be tonically con tracted also, causing elevation of the right shoulder and flexion of the right side of his neck. The twitchings were last noticed on Sept. 6th, but the tetanic rigidity remained undiminished up to the time of the patient's evacuation to England.

Throughout theie was cutaneous anæsthesia to pin-pricks and light touch over the area of distribution of the fourth cervical nerve.

Inowbation period.-The incubation period in the fatal cases varied from 6 to 11 days, the average being 8 days. in the non-fatal cases the incubation period varied from 11 to 22 days, the average being 16 , so that the prognostic significance of a short incubation period appears to be identical in local and general tetanus-that is to say, so far as there few cases indicate.

Duration. - The duration of the disease in the cases which recovered, estimated by the period during which twitchings were observed, was between three and four weeks. It is difficult to be more exact, because the twitchings did not suddenly cease; they gradually became less frequent, less easily evoked, and less violent, so that their final disappearance was not a striking event to which a definite date could be affixed. The tonic spasm continued unabated so long as the patients were under our care.

Mortality.-The mortality was 50 per cent. This seems a peculiar fact, especially so inasmuch as the patients did not die of exhaustion from general spasm or from respiratory spusm. Presumably they did not die of sepsis in the ordinary sense of the term, for one had a clean amputation stump, and another had a healed abdominal wound, as the only source from which such septic absorption could have been derived. On the whole, it seems probable that the cause of deati $\hat{K}_{1}$ was tetanus toxæmia, causing a medullary paralysis, though I have not, seen any prezious reference to this mude of death from tetanus.

\section{Theoretioal Considerations.} joint was septic, and was treated by incisions and fixation. On July loth twitching began in the left leg and foot. On the following day the right leg and foot were also involved, and amputation was performed through the left thigh. Three
In discussing the subject of local tetanus a great many points of interest will arise. For local tetanus, as has beev 
stated already, is a new form of disease. It is tetanus modified by the prophylacric injec'ion of antitetanus serum. And it is the general adoption of this prophylactic treatment that has given us the opportunity to study the conditions. Cases of tetanus beginning locally and followed by tri-mus and general spasms have long been described, but pure local tetanus is a new thing so far as man is concerned. It is not unknown in animals. In the cat, which is relatively insusceptible to telanus, a sub-fatal dose of tetanus toxin prodnces a persistent tonic spasm of the injected limb, and nothing else. And the same is true of the guinea-pig, if the dose of toxin be immediately followed by a sufficient dose of antitoxin. From which facts we may assume that the localisation of tetanus in man, as in the cat and guinea-pig in these experiments, is due to the presence of tetanus antitnxin in the blood.

We have still to consider why, with antitoxin circulating in the blood, there should develop any tetanus at all, and the solution of this question has been provided by experiments on animals. These experiments show that there are two avenues of approach whereby the toxin of tetanus may gain access to the central nervous system. These two Evenues are: (1) The general blood stream; (2) the motor nerves. If both these avenues are open general tetanus will ensue. If both are closed, there will be no tetanus. If No. 1 is closed while No. 2 in open, local tetanus will result. Further, the route by the general blood stream is more easily barred than is that by the motor nerves. For toxin circulating in the general blood stream is readily neutralised by antitoxin present in the same medium. whereas the toxin, once it has gained entrance to a motor nerve, is almost insusceptible to the neutralising action of antitoxin. And it has been shown also, that when tetanus toxin is injected into a muscle, its passage into the nerve is rapid, so rapid tht it may forestall the antitoxin and bring abuut local tetanu. Incidentally, it would seem that local tetanus is not likely to occur at all unless there is direct injury of muscle tissue-that is to say, if the hypothesis of causation is correct, local tetanus is unlikely to follow. for ex rmple, a wound involving skin and subcutaneous tissue only. Observations on this point are desirable.

Some hold ${ }^{6}$ that the peripheral nerves are the sole means of entry of tetanus toxin to the central nervous system, and they explain the absence of generalised tetauus after subcutaneous administration of antitetanus serum as due to immunisation of the higher centres thereby, this immunisation being insufficient to overcome the relatively concentrated attack on the cord at the place of origin of the motor nerves. So far as treatment is concerned. it is immaterial whether this or the foregoing hypothesis is the correct one.

Can Tetanus following Gunshot Wound be Abolished?

General experience has shown that a great diminution in the occurrence of tetanus has followed the systematic use of antitetanus serum in cases of gunshot wound. There is strong evidence that it can be aboli-hed, provided that two conditions are fulfilled. The two conditions are:-

(1) Firstly, that the initial dose of antitetanus serum is given sufficiently soon after the infliction of the wound. It has been shown that once the toxin has entered the motor nerves, or the central nervous system, it is not readily neutralised by antitoxin, and while we may suppose that tetanus bacilli require a little time to become established in a wound and to produce a material amount of poison, it also requires a certain period of time for antitoxin, injected subcutaneously, to produce much effect. It is stated ${ }^{7}$ that with subcutaneous injections of tetanus antitoxin, as with similar injections of antidiphtheritic serum, two days are required for a maximum effect.

(2) The second condition is that the dose must be repeated. Tetanus antitoxin does not persist indefinitely in the blood. It disappears rather rapidly-ten days is given as its average time of endurance-and therefore the dose must be repeated at intervals of a week or thereabout, to ensure the maintenance of a proper amount of antitoxin in the blood. For by injecting antitoxin we do not dentroy the tetanus bacilli in a wound. These continue to flourish, and to manufacture their toxin in spite of it, and have been found in a vigorous and virulent condition for as long as six meeks after the wound was received ${ }^{8}$

Duration "f effint of antitnxin - Ten days have been given as the period during which a single dose of antitoxin is effective. This, of course, is a general rule only. In the third case of local tetanus (Case 5) the patient had been given a dose of antitoxin nine days before receiving the wound from which modified tetanus developed, and 23 days before the first symptoms appeared. From which it appears that antituxin in some cases mav remain potent in the blood for more than ten days; potent, that is to say, to prevent general tetanus. Perhaps it may last a great deal longer than this, as the following case seems to indicate.

A man was wounded in the foot in November, 1915, and was given antitetanus serum. On July 2nd, 1916, he received slight wounds in the back of his neck. $\mathrm{He}$ remained with his battalion, and no serum was given. On Sept. 14th stiffness of the jaws and difficulty of swallowing developed, for which he was treated at a casualty clearing station. These symptoms lasted for about ten days, and then passed off without the development of any grave consequences. Presumably this was a case of modified tetanus, although eight months had elapsed between the injection of antitetanus serum and the receipt of the second wound, and ten months between the injection and the flrst appearance of symptoms.

I have not inclurled this among our cases of local tetanus, because the chief symptoms had disappeared by the time that we admitted him.

On the other hand, there is clear evidence that not only local but general tetanus may in some instances follow a gunshot wound, in spite of a single prophylactic dose of antitetanus serum.

We had such a case in 1915, in which general tetanu developed 21 days after a gunshot wound of the forearm, although a prophylactic dose of antitetanus serum had been anministered shortly af ter the receipt of the wound. This patient, an officer, was a chemist in civil life, and was absolutely sure that an initial prophylactic dose of serum had been given to him.

Site of injection. - Judging from the knowledge gained by experiments on animals, there seems to be some advantage from injecting the serum in the first instarce into the muscles of the wounded limb. This certainly seems to be advisable in cases where, for any reason, delay has come about in giving the initial dose ; for example, in the case of a wounded man who has lain out for some days after being wounded.

\section{Treatment of Established Tetanus.}

In a case of established tetanus we must remember that, although the symptoms are local at first, they may become general. And, therefore, for purposes of treatment we cannot always differentiate between local and general tetanus, and we are not likely to be wrong if we assume that the patient is threatened with the greater evil. And this is so, even though it be stated on the patient's field medical card, that antitetanus serum has been given. For the administration of these prophylactic doses is often carried ont by others than qualified medical men, a circumstance which is unavoidable, and yet carries with it the possibility of technical error. Moreover, as shown above, general tetanus may sometimes follow in spite of the proved use of prophylactio treatment.

The indications are: 1 . To neutralise the toxin which is present in the circulating blood. 2. To neutralise the toxin which is passing into the nerves of the injured part. 3. To nentralise as soon as possible the toxin which has already reached he central nervous system.

Subcutaneous injection is too slow in its action to effect any of these objects, for speed is of urgent importance, and if no time is to be lost at all it will be necessary to employ three methods of introduction-namely, (1) intravenous; (2) intramuscular, i.e., into the muscles of the affected limb; (3) intrathecal. A suitable treatment would be the immediate intravenous injection of 3000 units of antitetanus serum, accompanied by the introduction of similar amounts in to the spinal theca and muscles of the affected limb.

\section{Anaphylaxis.}

What dangers are to be feared from anaphylaxis? Personally, I believe that unless precautions are taken there is a real danger-in the case of intravenous injections, at any rate, of causing anaphylactic shock by secondary doses. I believe caces of sudden death to he examples of anaphylactic shock and to indicate that the secondary injection of antitoxin into a vein, in the presence of anaphy laxis, is a dangerous procedure unless certain precautions be taken. 
Reoognition of anaphylaxis.-How can we know if anaphylaxis is present? We can assume its presence if the patient has had a dose of serum ten or more days previously. As to the length of time during which anaphylaxis will continue there seems to be no definite information. Nevertheless, as anaphylaxis is present in some individuals to whom no serum has been administered previously, it is necessary to take special precautions in all cases in which the intravenous Foute is üsed for the injection.

By the following method danger from anaphylactic shock is said to be avoided. ${ }^{9} 5$ c.c. of antitoxic serum are diluted with 50 c.c. of warm saline. 1 c.c. of this is run into a vein. 4 minutes later 3 c.c. are run in; 2 minutes later 10 c.c., and 2 minutes later again 25 c.c. are run in, and after a pause of 10 minutes the remainder is injected. After this the intrathecal and intramuscular injections may be made without further special precaution against anaphylactic shock.

For some hours following the performance of intrathecal injection of antitetanus serum it is necessary, of course, to keep the foot of the patient's bed well raised. We know, from experience with spinal anæsthesia, that fluid injected into the lower portion of the spinal theca will not travel up the cord if the patient's dorsal spine be raised above the level of his lumbar spine. We also know that the fluid will travel up if the foot of the bed be raised. And what is true for a solution of novocain we may assume to be true of a solution of tetanus antitoxin.

Besides the administration of antitetanus serum, other therapeutic measures need consideration. Of drugs, chloral, bromide, and morphia have been the most used. For procuring rest paraldehyde in large doses has seemed of great benefit, and is perhaps more effective than any other drug. In severe cases a watch must be kept for acidosis, which is said to be present, as might be expected, in most of these. ${ }^{10}$

Treatment of the wound also is of importance. The natural impulse is to operate at once in order to eradicate the offensive bacilli, to excise necrotic tissue, to remove foreign bodies, and to provide free drainage; or to amputate the limb, but caution is required here, for experience has shown that operations on wounds infected with tetanus bacilli are likely to bring about a sudden diffusion of toxin, with consequent disaster. And the same is true of amputation, even though the amputation be performed before tetanus has declared itself. Operations, therefore, should be deferred, if possible, in all cases of established tetanus until sufficient time has passed for artificial immunisation to have been effected.

Immobilisation, however, so far as this is possible, is of paramount importance. We know the ill-effect of movement in the case of wounds infected with tuberculosis or the organisms of sepsis, an effect which consists of diffusion of toxins and possibly of bacteria also. A similar effect ensues in the case of wounds infected with the bacilli of tetanus. And not only the wound but the patient also, so far as is possible, must be kept at rest. In general illustration of this point may be quoted a case that we had in 1915.

The patient, an officer, had passed successfully through an attack of general tetanus. After the symptoms had subsided and we regarded him as cured he was evacuated to England. On his arrival there tetanus again developed, and he underwent a second attack, brought on, there is little doubt, by the disturbances of his long journey.

\section{Conclusion.}

In conclusion, I should like to ask the following open questions :-

1. What cases of modified tetanus have been met with ?

2. What have been the special features?

3. Have areas of cutaneous anæsthesia been observed in these cases?

4. Has persistent tetanic rigidity been present?

5. What is the cause of death in fatal cases?

6. Have any cases of anaphylactic shock been observed, and, if so, in what circumstances?

7. Have any cases of sensory tetanus been observed?

Answers to these questions I hope will be forthcoming, and also a great deal of other information on this interesting subject of local tetanus. If we are to understand the condition thoroughly, we must learn about it now, while there are cases available for study. It is to be hoped, therefore, that medical men who have had cases under their care will forthwith publish the details, and so communicate to others the benefit of their own experiences.

continued at foot of next column.

\section{W EIL'S DISEASE (SPIROCHATOSIS ICTERO-H AMMORHAGICA) IN THE BRITISH ARMY IN FLANDERS.*}

\author{
By A. STOKES, M.D. DuB., F.R.C.S. IREL.,
} CAPTAIN, ROYAL ARMY MEDICAL CORPS;

\author{
J. A. RYLE, M.B., B.S. LoND.,
}

CAPTAIN, ROYAL ARMY MEDICAL CORPS, SPECIAL RESEREE, $\triangle N D$

W. H. TYTLER,

captain, oanadian aRmy medical corps.

A SHORT preliminary note ${ }^{1}$ on infectious jaundice among British troops in France was written in the early summer of 1916. Although the investigation of this disease is still being pursued both here and elsewhere and is as yet far from complete, the accumulation of considerably more material, both clinical and experimental, since our last publication would seem to justify a further communication at the present moment. The additional results which we have attained contain little not already described by Inada and his collaborators, but they seem of interest in that our confirmation of the findings of the Japanese workers, at the time of our first note only partial, has now been rendered almost complete. The only results which we have not been able to duplicate are their success in growing the causal organism in artificial culture and the finding of spirochætæ in the urine. The possible explanation of the former point and the evident explanation of the latter are discussed below.

\section{Previous Work.}

The disease commonly bears the name of Weil, who described it in 1886, but Martin and Pettit, in a recent publication discussed below, point out that this is quite unjust in that several French authors, quoted from Kelsch (1894), had previously described the condition under various names, and at dates extending from the siege of Cairo down to 1886. Those quoted are: Larrey, Ozanam (1849), Monneret (1859), Laveran the elder(1865), Lancereaux (1882), Landouzy (1882), and Mathieu (1886).

Weil described the chief characteristios of the disease as being jaundice, pyrexia, and hæmorrhages, and the occurrence of cases in epidemics or localised groups. Widespread epidemics have been recorded in the United States, and also outbreaks in India, Africa, and the Near East. In Osler's Medicine we find:-

The symptoms are at first gastric, then fever follows (with the usual concomitants) and jaundice, which may be slight or very intense, and, as a rule, albuminuria. The liver and spleen are often enlarged, and in the severe forms there are nervous symptoms and hømorrhages. There is often a secondary fever, and the atacks last from ten days to three weeks. The course is usually favourable; fatal cases are rare in the United States and in India and South Africa, but in the Greek hospital at Alexandria the death-rate was 32 among 300 .

No definite etiology had been established until the subject of infectious jaundice was reopened by a monograph from Drs. Inada, Ido, Oki, Kaneko, and Ito, which was first published in the English language in March, 1916, in the Journal of Experimental Medioine. In this paper the authors state :-

In the western part of Japan there prevails an epidemic and endemic disease, characterised by conjunctival congestion, muscular pains, fever, jaundice, hæmorrhagic diathesis and albuminuria, which is known as Weil's disease or febrile jaundice. At the end of last year (1914) the same disease was observed in Shiba, in the eastern part of Japan near Tokio, where the patients numbered 178.

* Received for publication on Dec. 8th, 1916.

1 Journal of the R.A.M.C., September, 1916, and Brit. Med. Jour., Sept. $23 r d, 1916$.

Continued from preceding column.

References.-Montais and Broch : Annales de l'Institut Pasteur, July, 1916, quoted in THE LANCE', 1916, i1., 834. 2. Courtois-Suffit and Giroux : Les Formes Anormales du Tétanos, 1916. 3. Lne. eit. 4. Loc. cit. 5. Montais and Broch: Loc. cit. 6. Burnet and Tulloch : Brit. Jour. Surg., July, 1916. 7. Browning: Brit Jour. Surg., July, 1916.
8. Robertson: Lister Institute, Nov. 23rd, 1916. 9. Browning : Loc. oit. 10. Embleton: Trans. Med. Soc, Lond., Xxxviii.; 